\title{
Amiodarone Induced Organizing Pneumonia: A Masquerader of Community Acquired Pneumonia
}

\author{
Ana Vigário* and Catarina Mendonça \\ Hospital Centre of Oporto, Santo António Hospital, Internal Medicine Service, Oporto, Portugal
}

\begin{abstract}
Amiodarone is a widely used antiarrhythmic with well-known adverse effects, being pulmonary toxicity one of the most serious, occurring even with low doses. Amiodarone-induced organizing pneumonia (AIOP) is an infrequent histopathological presentation but a few cases have been reported in the literature.

A 78-year-old woman with atrial fibrillation doing amiodarone $200 \mathrm{mg}$ once daily for two years was admitted to the Emergency Department with progressive dyspnea to rest, productive purulent cough, flulike symptoms and pleuritic chest pain. Blood tests revealed a hypoxemic respiratory failure and a systemic inflammatory response, and the chest radiography showed bilateral, multifocal pulmonary infiltrates. Microbiologic studies of urine, blood and sputum were sterile. The patient was initially treated for community acquired pneumonia, with clinical and radiological worsening despite multiple extended spectrum antibiotics. The complementary study excluded other etiologies, and a transthoracic pulmonary biopsy was performed, revealing histology consistent with organizative pneumonia.

AIOP is typically presented as a community acquired pneumonia that does not respond to antibiotics. The diagnosis depends on a high clinical suspicion, and compatible clinical and radiological pattern. Although it is not the most frequent, the presented case enforces the assumption that toxicity is present even with lower doses such as 200 $\mathrm{mg}$ once daily for 2 years.
\end{abstract}

Keywords: Amiodarione; Pulmonary toxicity; Organizing pneumonia; Treatment

\section{Introduction}

Amiodarone is one of the most common antiarrhythmic medications prescribed worldwide [1] and its adverse reactions are very frequent (75\% of patients) [2] and extensively studied: corneal deposits in over $90 \%$ of the patients (and also development of cataract and optic neuropathy), interaction with other cardiac drugs, symptomatic bradycardia, hepatotoxicity, gastrointestinal disturbances, dermatitis, bluish discoloration of the skin, bone marrow suppression, coagulopathies, peripheral neuropathies, hyperthyroidism and hypothyroidism, being thyroid dysfunction the predominant one [1,3-5].

After the introduction of amiodarone as an antiarrytmic in 1967, pulmonary toxicity was first described in 1980 [6] and has been reported in some series as the third most common reaction [3], being among the most severe [5]. It is present in $5-10 \%$ of patients taking amiodarone [5-8] and it can occur at any dose, even with low dose therapy [9]. The risk for toxicity increases with higher plasma concentrations [10]. However, Amiodarone pulmonary toxicity (APT) is better correlated with the total cumulative dose, rather than with the daily dose or plasma concentration of amiodarone [10,11]. Toxicity can occur at any time after treatment has been initiated. However, it has been described that those who have received a daily dose of $400 \mathrm{mg}$ or more, for more than two months, or a lower dose, commonly $200 \mathrm{mg}$ daily, for more than 2 years are at greater risk $[11,12]$. The initial loading dose is also believed to be an important contributor as it significantly increases the cumulative dose [4].

Amiodarone and its metabolites can produce lung damage directly by a cytotoxic effect, and indirectly by an immunologic reaction $[11,13]$. The most typical clinical and histopathological presentation is an interstitial pneumonitis, but less frequent presentations have also been described as such organizative pneumonia and diffuse alveolar damage [14-17].

\section{Report}

A 78-year-old woman with a history of hypertensive cardiopathy and atrial fibrillation was admitted with a progressive dyspnea to rest, productive purulent cough, nocturnal wheezing, flu like symptoms and pleuritic chest pain, over one week. She also complained with dry cough and dyspnea on exertion for the past 6 months. She was on losartan/ hydrochlorothiazide 50/12.5 mg od, acetylsalicylic acid $100 \mathrm{mg}$ od, and she had also been taking amiodarone $200 \mathrm{mg}$ od for the last 2 years (cumulative dose $146 \mathrm{~g}$ - initial loading dose unknown).

On examination she presented with hemodynamic stability (arterial blood pressure 122/71 $\mathrm{mmHg}$; heart rate $89 \mathrm{bpm}$ ), polypnea to minimum effort with a respiratory rate of $24 \mathrm{cpm}$, and bilateral inspiratory crackles, predominant in the right hemi thorax. There was no neurologic impairment. Blood tests revealed hypoxemic respiratory failure $\left(\mathrm{pO}_{2} / \mathrm{FiO}_{2} 232\right)$ and systemic inflammatory response. The chest radiography displayed a bilateral, multifocal pulmonary infiltrates (Figure 1). Microbiologic studies of urine, blood and sputum were sterile. She was initially treated for community acquired pneumonia, with clinical and radiological worsening despite multiple extended spectrum antibiotics. High-resolution computed tomography (HRCT) showed diffuse parenquima consolidation, with predominance in inferior lobes and posterior right superior lobe; and diffuse pericentimetric ganglionar formations and in subcarinal region (Figures 2-4). Bronchofibroscopy excluded local malignancy and the pathology of bronchoalveolar lavage (BAL) showed inflammatory infiltrates without granulomas or malignant cells. BAL immunology study was negative for alveolitis with $1,86 \times$

${ }^{*}$ Corresponding author: Ana Sofia Coutinho Vigário Rodrigues, Hospital Centre of Oporto, Santo António Hospital, Internal Medicine Service, Oporto-4050-637, Portugal, Tel: 00351 913091451; E-mail: anavigaria@gmail.com

Received January 20, 2016; Accepted January 27, 2016; Published January 31, 2016

Citation: Vigário A, Mendonça C (2016) Amiodarone Induced Organizing Pneumonia: A Masquerader of Community Acquired Pneumonia. J Pulm Respir Med 6: 319. doi:10.4172/2161-105X.1000319

Copyright: @ 2016 Vigário A, et al. This is an open-access article distributed under the terms of the Creative Commons Attribution License, which permits unrestricted use, distribution, and reproduction in any medium, provided the original author and source are credited. 
Citation: Vigário A, Mendonça C (2016) Amiodarone Induced Organizing Pneumonia: A Masquerader of Community Acquired Pneumonia. J Pulm Respir Med 6: 319. doi:10.4172/2161-105X.1000319

$10^{6}$ cells, with lymphocyte predominance of $41 \%, 18 \%$ neutrophils, and $12 \%$ eosinophils, with a low CD4/CD8 of 0,157 , making the hypothesis of interstitial pneumonitis remote. BAL microbiological, mycobacteriological and mycological studies were negative, as well as HIV-1/2, therefore infectious etiology was extremely unlikely. ACE was negative, CD4/CD8 was low and there was no hypercalciuria, excluding sarcoidosis. It was performed a transthoracic pulmonary biopsy, which revealed an increased thickness of the septae, and a fibro-edematic nodule (appearing in one of the represented air spaces), corresponding to an intra-alveolar polyp, consistent with organizative pneumonia. Granulomatose lesions were absent.

Giving the unfavorable clinical course, and the extensive investigation excluding other pathological entities, namely infectious causes, it was considered the diagnosis of AIOP.

Thyroid function was normal. Fundoscopy was performed, showing bilateral whorl-like pattern of corneal epithelial deposits, consistent with amiodarone-induced vortex keratopathy, also called cornea verticillata, markers of amiodarone exposure [18].

Amiodarone was stopped and systemic corticoid therapy initiated with prednisolone $1 \mathrm{mg} / \mathrm{Kg} /$ day. There was an impressive clinical response with complete resolution of symptoms and respiratory failure in ten days. Imagiological reevaluation with HRCT at D12 showed significant reduction in bronchopneumonic consolidations and a discrete reduction in mediastinal ganglia. Respiratory functional tests were performed only at D15 of prednisolone, revealing obstruction of small airways, but no restriction pattern and normal DLCO corrected to lung volumes. Prednisolone was tapered gradually, and discontinued 10 months later, with complete clinical and imagiological resolution (Figures 1-7).

\section{Discussion}

Organizative pneumonia is commonly presented as community acquired pneumonia, with progressive dyspnea, cough, flu like symptoms, and a typical radiographic pattern mostly with alveolar, multifocal, irregular consolidations [19], being irresponsive to several antibiotic courses, as occurred in this case.

About $50 \%$ of occurrences correspond to a cryptogenic form, with the remaining having its origin in connected tissue diseases, drugs (amiodarone, carbamazepine, cephalosporin, nitrofurantoin, phenytoin, etc.) and infections (bacterial such as mycoplasma

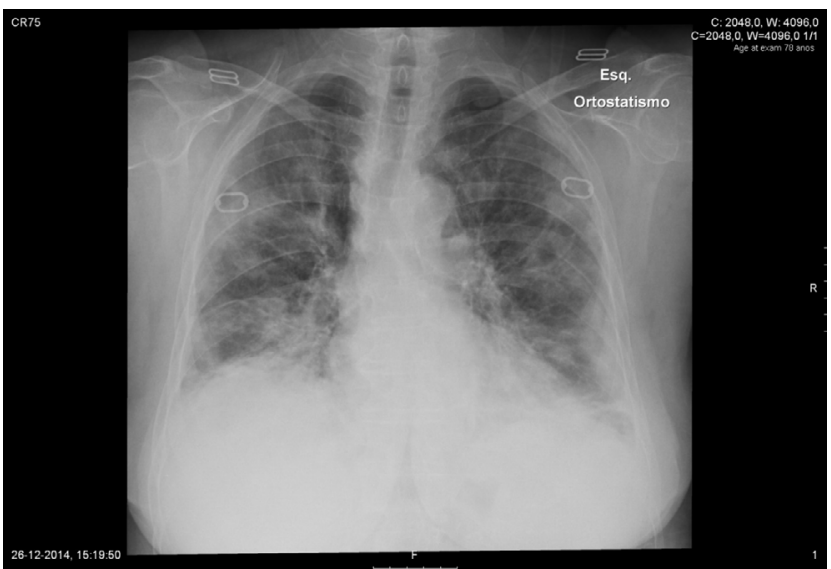

Figure 1. Chest radiography showing a bilateral, multifocal pulmonary infiltrates.

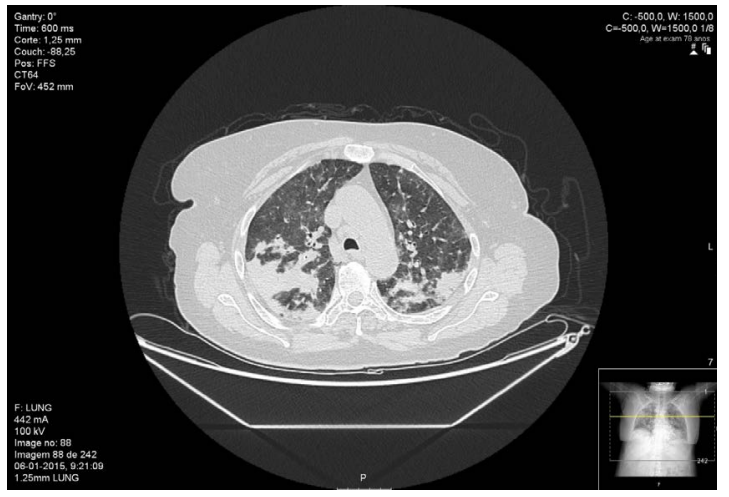

Figure 2: HRCT showed diffuse parenquima consolidation, with predominance in inferior lobes and posterior right superior lobe; and diffuse pericentimetric ganglionar formations and in subcarinal region.

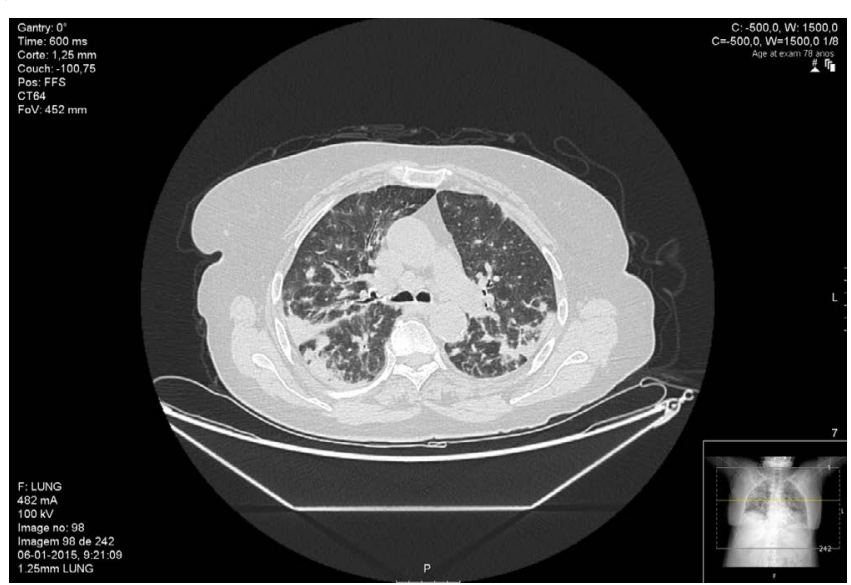

Figure 3: HRCT showed diffuse parenquima consolidation, with predominance in inferior lobes and posterior right superior lobe; and diffuse pericentimetric ganglionar formations and in subcarinal region.

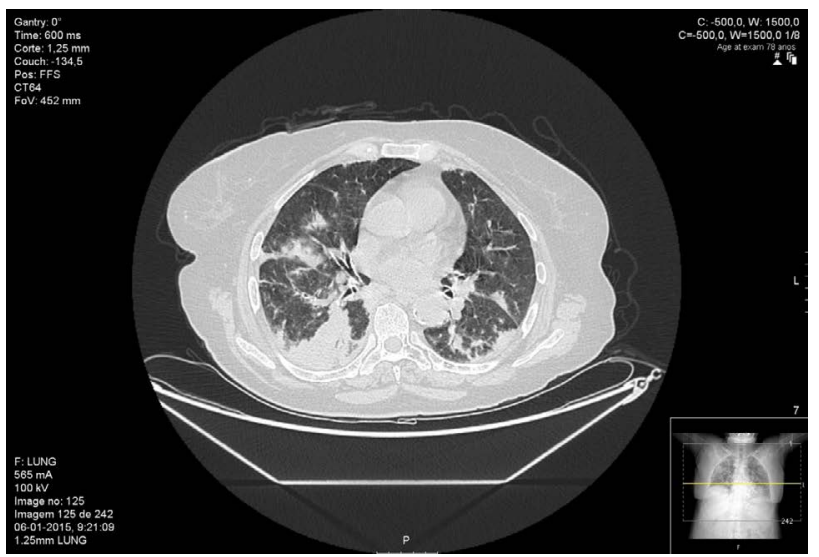

Figure 4: HRCT showed diffuse parenquima consolidation, with predominance in inferior lobes and posterior right superior lobe; and diffuse pericentimetric ganglionar formations and in subcarinal region.

pneumonia, legionella, pseudomonas aeruginosa, streptococcus pneumonia, staphylococcus aureus, but also viral: HIV, Herpes simplex, influenza; fungi: Cryptococcus neoformans, Pneumocystis jiroveci; and parasites: plasmodium vivax) $[19,20]$.

The diagnosis depends on a high clinical suspicion, and compatible 


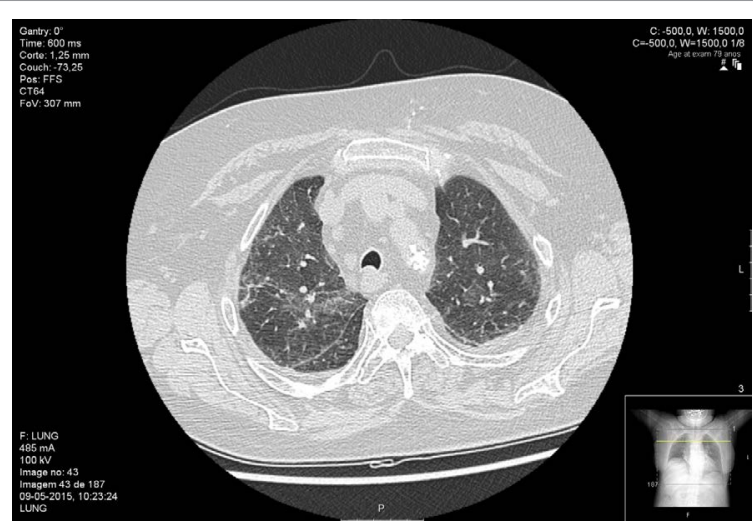

Figure 5: HRCT documenting imagiological response after 3 months of treatment.

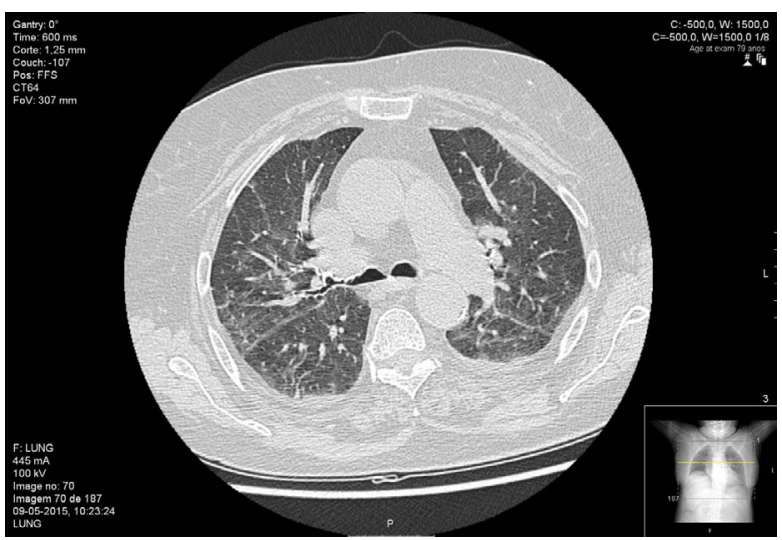

Figure 6: HRCT documenting imagiological response after 3 months of treatment.

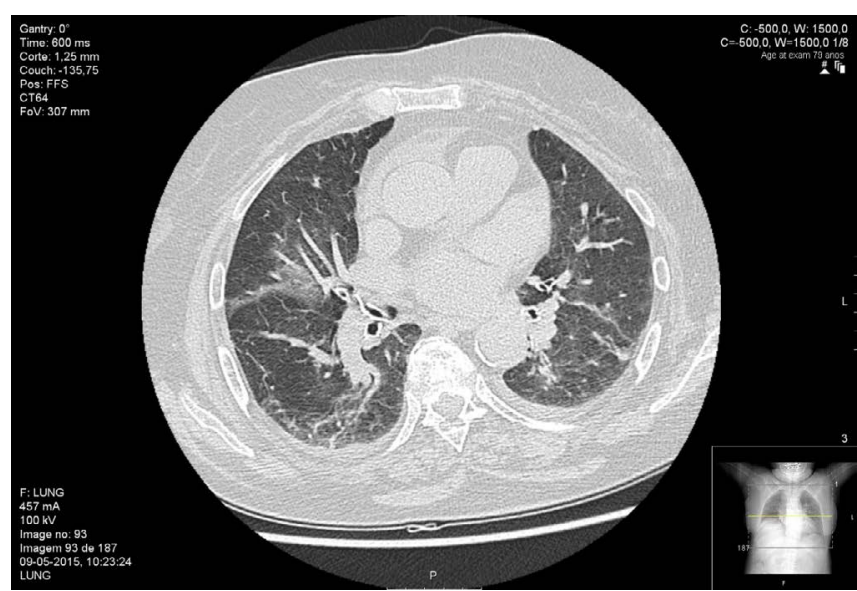

Figure 7: HRCT documenting imagological response after 3 months of treatment.

clinical and radiological pattern. A restrictive pattern in PFTs and a reduced DLCO (>15-20\%) strengthens the diagnosis [5,11,20,21]. It may be confirmed with typical histopathological features from lung biopsy, rather obtained by video assisted thoracoscopy (VATS) or open thoracotomy, but it is acceptable and usually more reasonable to perform transbroncquic biopsy, especially in those with typical clinical and radiological features[19,20]. In this case only transthoracic biopsy was performed but the typical clinical presentation, the exposure to amiodarone and the exclusion of other etiologies makes our hypothesis likely.

Risk factors for APT have been suggested such as daily dose, cumulative dose, duration of therapy, patient age and the presence of pre-existing lung disease $[3,5,11]$, but this is not consensual and more investigation is needed in order to define which risk factors are preponderant in development of AIPT to identify the patients at greater risk needing closest monitoring. D.K. Ernawati et al. tested the first four in a large database, and only patient age and duration of amiodarone therapy were confirmed as significant risk factors [3].

AIPT is always a diagnosis of exclusion [5], and there is no way of proving a cause-effect. The exposure risk factor and the typical clinical and imagiological patterns are supporting the diagnosis.

PFT usually reveals low lung volumes, a restrictive pattern, and a reduced DLCO. The lack of these patterns in this patient may be explained by the delay in the availability of PFT that were deferred for two weeks later under corticosteroids. An obstruction of the small airways was documented in this case, a defect that has also been described $[5,11,20,21]$.

Treatment decision is made considering disease severity. Stopping amiodarone is mandatory, and it may be sufficient in milder cases, with monitoring of lung function. It is recommended the use of oral corticosteroids in persistent or gradually worsening disease, which usually provide a rapid improvement of symptoms, as it did in our patient $[5,11,21]$.

In case of fulminant disease, intravenous glucocorticoids should be used, with metilprednisolone $125-250 \mathrm{mg}$ each $6 \mathrm{hrs}$ or $750-1000 \mathrm{mg} /$ day for 3 to 5 days. In the other cases it is suggested an initial dose of prednisolone of $0.75-1 \mathrm{mg} / \mathrm{Kg} /$ day, (to a maximum of $100 \mathrm{mg} /$ day). This dose should be maintained for 4 to 8 weeks, and then gradually tapered to $0.5-0,75 \mathrm{mg} / \mathrm{Kg} / \mathrm{day}$ for 4 to 6 weeks. After 3 to 6 months, the dose of oral prednisolone should be gradually tapered to zero if the patient remains stable or improved $[20,22]$. The patient should be routinely monitored with chest radiography and PFT every 2 to 3 months while under glucocorticoid therapy [20]. Relapses are common [19], but it has not occurred in our case 10 months later. It usually occurs when glucocorticoids are tapered, after 1 to 3 months $[19,20]$. Most of these patients will improve, when going back on prednisolone and the occurrence of relapses does not seem to affect the long term prognosis [22]. After the suspension, the patient should be monitored in the following year with a chest radiograph every 3 months [20].

A monitoring protocol has not been stablished worldwide, but some authors have suggested complete pulmonary function testing (PFT), including a diffusing capacity for carbon dioxide (DLCO) and chest radiography, before initiation of amiodarone therapy, every 3 months in the first year, yearly thereafter, and whenever pulmonary toxicity is suspected $[2,21]$.

\section{Conclusion}

AIOP is typically presented as community acquired pneumonia that does not respond to extended spectrum antibiotics. The diagnosis depends on a high clinical suspicion, and compatible clinical and radiological pattern.

Although it is not the most frequent, our case supports that toxicity is present even with lower doses, such as $200 \mathrm{mg}$ daily for 2 years. We must be aware of the multiple adverse reactions of amiodarone, and 
Citation: Vigário A, Mendonça C (2016) Amiodarone Induced Organizing Pneumonia: A Masquerader of Community Acquired Pneumonia. J Pulm Respir Med 6: 319. doi:10.4172/2161-105X.1000319

every physician should keep in mind that monitoring these patients is crucial. It is important to alert patients about the potential pulmonary toxicity, and the importance of reporting any respiratory symptom.

A monitoring protocol has not been approved yet, but it has been suggested that patients taking amiodarone should be tested with chest radiography and PFT: before starting treatment, every 3 months in the first year, yearly thereafter, or anytime they experience symptoms. A validated strategy is urgent to better anticipate these harmful effects.

\section{Key messages}

- AIOP typically presents as a community acquired pneumonia, with typical radiologic pattern and usually a restrictive pattern with reduced DLCO in PFT.

- It is important to have an elevated suspicion index.

- It is always an exclusion diagnose after ruling out other life threatening pulmonary diseases.

- Toxicity is present even with lower doses, such as $200 \mathrm{mg}$ daily for 2 years

- It is important to alert patients doing amiodarone about the potential pulmonary toxicity and the importance of reporting any respiratory symptom.

- Patients taking amiodarone should be submitted to chest radiography and PFT: before starting the treatment, every 3 months in the first year, yearly thereafter, or anytime they experience symptoms.

\section{Consent}

All authors declare that written informed consent was obtained from the patient for publication of this case report.

\section{Ethical Approval}

Not applicable.

\section{Competing Interests}

Authors have declared that no competing interests exist.

\section{References}

1. Vassallo P, Trohman RG (2007) Prescribing amiodarone: an evidence-based review of clinical indications. JAMA 298: 1312-1322.

2. Silva MN, Bacellar P, Martins H, Tinoco N, Costa F (2006) [Amiodarone induced pulmonary fibrosis--a clinical case report]. Rev Port Pneumol 12: 725-730.

3. Ernawati DK, Stafford L, Hughes JD (2008) Amiodarone-induced pulmonary toxicity. Br J Clin Pharmacol 66: 82-87.

4. Kudenchuk PJ, Pierson DJ, Greene HL, Graham EL, Sears GK, et al. (1984) Prospective evaluation of amiodarone pulmonary toxicity. Chest 86: 541-548.

5. Martin WJ 2nd, Rosenow EC 3rd (1988) Amiodarone pulmonary toxicity Recognition and pathogenesis (Part I). Chest 93: 1067-1075.

6. Rotmensch HH, Liron M, Tupilski M, Laniado S (1980) Possible association of pneumonitis with amiodarone therapy. Am Heart J 100: 412-413.

7. Dusman RE, Stanton MS, Miles WM, Klein LS, Zipes DP, et al. (1990) Clinica features of amiodarone-induced pulmonary toxicity. Circulation 82: 51-59.

8. Schwaiblmair M, Berghaus T, Haeckel T, Wagner T, von Scheidt W (2010) Amiodarone-induced pulmonary toxicity: an under-recognized and severe adverse effect? Clin Res Cardiol 99: 693-700.

9. Ott MC, Khoor A, Leventhal JP, Paterick TE, Burger CD (2003) Pulmonary toxicity in patients receiving low-dose amiodarone. Chest 123: 646-651.

10. Rotmensch HH, Belhassen B, Swanson BN, Shoshani D, Spielman SR, et al. (1984) Steady-state serum amiodarone concentrations: relationships with antiarrhythmic efficacy and toxicity. Ann Intern Med 101: 462-469.

11. Wolkove N, Baltzan M (2009) Amiodarone pulmonary toxicity. Can Respir J 16: $43-48$

12. Camus P, Martin WJ 2nd, Rosenow EC 3rd (2004) Amiodarone pulmonary toxicity. Clin Chest Med 25: 65-75.

13. Martin WJ 2nd, Rosenow EC 3rd (1988) Amiodarone pulmonary toxicity Recognition and pathogenesis (Part 2). Chest 93: 1242-1248.

14. Valle J (1995) Bronchiolitis obliterans organizing pneumonia secondary to amiodarone: a rare aetiology. European Respiratory Journal 8: 470-471.

15. Gulmini LAS, Pereira CAC, Coletta EN (2001) Pneumonia em organização secundária ao uso de amiodarona. J Bras Pneumol 27: 167-170.

16. Camus P, Lombard JN, Perrichon M, Piard F, Guérin JC, et al. (1989) Bronchiolitis obliterans organising pneumonia in patients taking acebutolol or amiodarone. Thorax 44: 711-715.

17. Schindler K, Schima W, Kaliman JF (2010) Cryptogenic organizing pneumonia due to amiodarone: long-term follow-up after corticosteroid treatment. Wien Klin Wochenschr 122: 511-514.

18. Chan TC, Jhanji V (2015) Images in clinical medicine. Amiodarone-induced vortex keratopathy. N Engl J Med 372: 1656.

19. Cordier JF (2006) Cryptogenic organising pneumonia. Eur Respir J 28: 422 446.

20. King TE Jr (2015) Cryptogenic organizing pneumonia. 2015.

21. Magro SA, Lawrence EC, Wheeler SH, Krafchek J, Lin HT, et al. (1988) Amiodarone pulmonary toxicity: prospective evaluation of serial pulmonary function tests. J Am Coll Cardiol 12: 781-788.

22. Bradley B, Branley HM, Egan JJ, Greaves MS, Hansell DM, et al. (2008) Interstitial lung disease guideline: the British Thoracic Society in collaboration with the Thoracic Society of Australia and New Zealand and the Irish Thoracic Society. Thorax 63 Suppl 5: v1-58. 